\title{
Current-Voltage Characteristics of Nanowires Formed at the $\mathrm{Co}-\mathrm{Ge}_{99.99} \mathrm{Ga}_{0.01}$ Interface
}

\author{
M. WAWRZYNIAK ${ }^{a, *}$, M. MAĆKOWsKi $^{a}$, Z. ŚNIADECKI $^{b}$, B. IDZIKOWSKI ${ }^{b}$ \\ AND J. MARTINEK ${ }^{b}$ \\ ${ }^{a}$ Faculty of Electronics and Telecommunications, Poznań University of Technology \\ Piotrowo 3A, 60-965 Poznań, Poland \\ ${ }^{b}$ Institute of Molecular Physics, Polish Academy of Sciences \\ M. Smoluchowskiego 17, 60-179 Poznań, Poland
}

\begin{abstract}
We present a method of measurement of the current-voltage $(I-V)$ and conductance-voltage $(G-V)$ characteristics of nanowires with quantum point contact formed at the $\mathrm{Co}_{\mathrm{O}}-\mathrm{Ge}_{99.99} \mathrm{Ga}_{0.01}$ interface. The effect of the Fermi level pinning leads to the formation of an ohmic contact between Co and $\mathrm{Ge}_{99.99} \mathrm{Ga}_{0.01}$. On the measured characteristics, above the threshold value of voltage an exponential current growth is observed. Such effect could be useful in the production of the electronic nanodevices.
\end{abstract}

PACS numbers: 68.65.-k, 79.60.Jv, 73.63.Rt

\section{Introduction}

In recent years the significant development in the field of electronic systems of nanometers size built with the use of nanowires was achieved. Nanowires have been used to construct rectifying diodes [1, 2], electroluminescence diodes [3], $p-n$ transistors [1], field-effect transistors [4], chemical sensors [5, 6]. These devices encapsulate nanowires with $\mathrm{Si}$ [1], GaN [2], $\mathrm{ZnO}[3-5]$ created by various types of chemical synthesis. The diameters of nanowires used range from 20 [1] to $63 \mathrm{~nm}$ [5]. Further decrease of the nanowire diameter down to the value of the Fermi wave length leads to the creation of a quantum point contact (QPC) [7].

The conductance of a nanowire with the QPC is quantized with the conductance quantum $G_{0}=2 \mathrm{e}^{2} / h$ (where $e$ - elementary charge, $h$ - Planck's constant) that can be confirmed by step changes of conductance on time evolution plots (conductance traces) and maxima on conductance histograms $[8,9]$. To form a nanowire with a QPC a scanning tunneling microscope [7] as well as the mechanically controllable break junction (MCBJ) technique [10] are used. The specialized experimental setup allows measuring the $I-V$ characteristics of nanowires with QPC [11] in a short time range at particular conductance plateau. The $I-V$ measurements were carried out for $\mathrm{Au}[12,13], \mathrm{Pt}, \mathrm{Cu}$ and $\mathrm{Ag}[14]$ nanowires with QPC. The Au $I-V$ characteristics show nonlinearity for short nanowires, and linear behavior for long nanowires [15].

* corresponding author; e-mail: mwawrz@et.put.poznan.pl
Conductance-voltage measurements were also made for nanowires with QPC formed at metal-semiconductor interface [16]. In the latter case not only will the conductance quantizing effect have the influence on the electron transport but also an effect of the interface, which results mainly from the differences in electronic band structures of the metal and semiconductor creating the contact. For the semiconductors three processes are considered which cause an electric current flow: thermoelectrical emission, diffusion and tunneling. Possibility of creating a QPC in area of metal-semiconductor contact is a new interesting situation, because it is not clear which one of the mentioned physical mechanisms will have a decisive role in ballistic transport regime.

\section{Results and discussion}

The nanowires used in the experiments were formed between Co tip and the surface of a $\mathrm{Ge}_{99.99} \mathrm{Ga}_{0.01}$ alloy under atmospheric pressure and at room temperature. The alloy is polycrystalline $p$-type semiconductor with resistivity of about $\approx 4 \mathrm{~m} \Omega \mathrm{cm}$. The tip was made of cobalt wire with starting thickness $1 \mathrm{~mm}$ and $99.975 \%$ purity. The surface of $\mathrm{Ge}_{99.99} \mathrm{Ga}_{0.01}$ was etch polished with $\mathrm{CP} 4$ solution and next rinsed with deionized (DI) water.

According to the metal-semiconductor interface model, neglecting the surface electronic states, the ohmic contact emerges in two cases: (i) for a $n$-type semiconductor if $\Phi_{\mathrm{M}}<\Phi_{\mathrm{S}}$ and (ii) for a $p$-type semiconductor if $\Phi_{\mathrm{M}}>\Phi_{\mathrm{S}}$, where $\Phi_{\mathrm{M}}$ is a metal work function for electron and $\Phi_{\mathrm{S}}$ is a semiconductor work function. In both 
cases left, namely: $\Phi_{\mathrm{M}}>\Phi_{\mathrm{S}}$ for $n$-type semiconductor and $\Phi_{\mathrm{M}}<\Phi_{\mathrm{S}}$ for $p$-type semiconductor a rectifying contact is created. It is not enough to compare the work functions for Ge and metal in order to specify the type of the contact created between a Ge surface and a metal. The effect of the Fermi level pinning appearing in the interface between metal and $n$-type Ge causes the contact to be rectifying and rises the Schottky barrier the height of which is almost independent of the used metal work function $[17,18]$. On the other hand, due to the Fermi level pinning for $p$-type Ge, the contact of this semiconductor with metal show Ohmic behavior for all metals $[17,18]$.

A measurement setup with a digital oscilloscope was used to form the nanowires in the metal-semiconductor interface and to register the $I-V$ characteristics $[12,19]$. The nanowires are created between electrodes $A-$ a $\mathrm{Ge}$ sample and $B-$ a Co tip. Electrode $B$ was mounted to a piezoelectric actuator. In order to fabricate a nanowire, a collision between $A$ and $B$ electrodes is arranged. The next step is to push away electrode $B$ from electrode $A$ with the speed of $\approx 4 \mu \mathrm{m} / \mathrm{s}$. After the collision a permanent connection between the electrodes is created. Pulling of electrode $B$ causes the stretching of the area which took part in the collision. During this stretching nanowires are created. In the ending phase of the stretching between the electrodes a single nanowire remains. A QPC is formed right before the breaking of this nanowire in its thinnest place. During the pulling a nanowire is biased with a voltage of $0.4 \mathrm{~V}$. The oscilloscope registers two voltage signals which makes it possible to calculate both the value of the voltage bias in the nanowire and the electric current flowing though the nanowire during the pulling. In Fig. 1a examples of conductance traces are presented for nanowires formed between a Co tip and a $\mathrm{Ge}_{99.99} \mathrm{Ga}_{0.01}$ surface, which show step changes of conductance typical for the conductance quantization regime.

The effect of the step changes of the conductance caused by closing of the conductance channels results from decreasing of the QPC diameter in a nanowire while it is stretched. The thinnest area in a nanowire shows the properties of a QPC if its diameter is comparable to the Fermi wavelength of the material of the nanowire. The value of the Fermi level for cobalt is $E_{\mathrm{F}}(\mathrm{Co}) \approx 7.38 \mathrm{eV}$. We can estimate the Fermi wave length basing on the Fermi energy value, $\lambda_{\mathrm{F}}(\mathrm{Co}) \approx 0.45 \mathrm{~nm}$, that corresponds approximately to the diameter of a single Co atom. Taking this into account we can estimate that the width of the thinnest area in the nanowire creating QPC for Co corresponds to a single atom. The Fermi energy for Ge depends on a type of conduction ( $p$ or $n$ ) and doping concentrations. For Ge without doping the Fermi energy level lies in the center of the energy gap and is approximately $E_{\mathrm{F}}(\mathrm{Ge}) \approx 0.34 \mathrm{eV}$. For $n$-type doped $\mathrm{Ge}$ the Fermi energy level lies above that value and for the p-type doped Ge - below this value. After creation of the junction between the Co tip and the $n$-type Ge
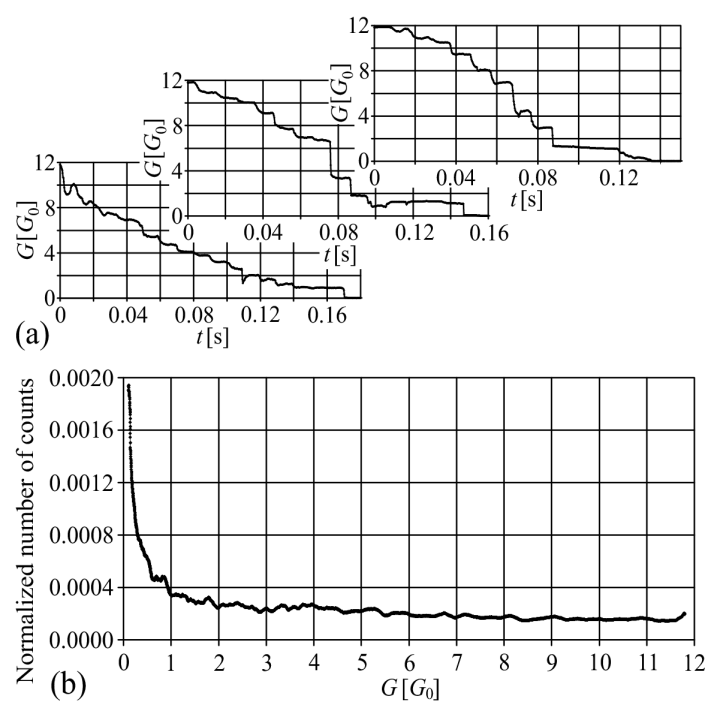

Fig. 1. Typical conductance traces (a) and a conductance histogram calculated on the basis of 2000 conductance traces (b).

or $p$-type Ge surface the Fermi energy level is strongly pinned near the charge neutrality level (CNL) [17, 20]. The CNL lies on level of $0.09 \mathrm{eV}$ [17] (0.08 eV [20]) above the higher valence band edge. If we assume that the Fermi energy level after connecting of the Co tip to the Ge surface lies at $0.09 \mathrm{eV}$, the estimated value of the Fermi wavelength in the system: Co tip-Ge surface will be $\lambda_{\mathrm{F}}(\mathrm{Co}-\mathrm{Ge}) \approx 4.09 \mathrm{~nm}$. For nanowires formed close to the $\mathrm{Co}-\mathrm{Ge}$ interface in nanowire a QPC will be created if the diameter of the nanowire in its thinnest area is of order $4 \mathrm{~nm}$.

In Fig. 1b a conductance histogram is presented, calculated on the basis of 2000 conductance traces [21]. The presented histogram does not show any clear maxima for particular values of conductance.

The results of the measurements of the $I-V$ characteristics for QPCs formed between a Co tip and a $\mathrm{Ge}_{99.99} \mathrm{Ga}_{0.01}$ surface are presented in Fig. 2a $(V-$ bias voltage, $I-$ current through the nanowire). Three typical characteristics are chosen which are measured for different points on the $\mathrm{Ge}_{99.99} \mathrm{Ga}_{0.01}$ surface in which a nanowire was formed. In Fig. $2 \mathrm{~b}$ the nanowire conductance $G=I / V$ as a function of bias voltage $V$ $(G-V)$ is presented, calculated on the basis of characteristics from Fig. 2a. The sweep of the bias voltage $V$ for nanowire with QPC during the measurement of the current-voltage characteristic is presented in the inset of Fig. 2a. Up to point 1, the nanowire is biased with constant voltage. The change of the bias voltage (from point 1) is triggered by the measuring set after it detects a plateau in the conductance at the level $G$, for which the $I-V$ characteristic is taken. After the triggering, an introductory stage before the measurement of the characteristic occurs between point 1 and 2. Voltage $V$ changes in range from $0.4 \mathrm{~V}$ to $V_{\min }$ (the lower edge of the mea- 


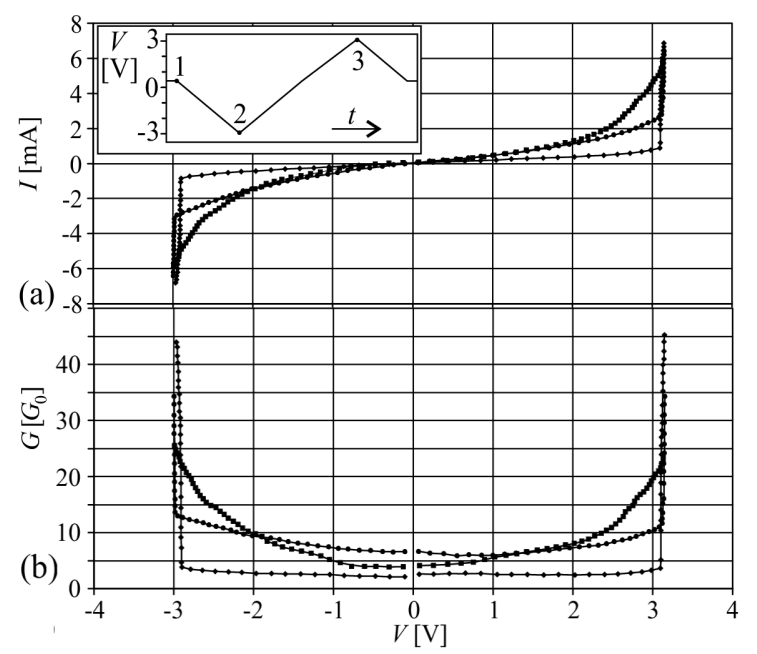

Fig. 2. $I-V$ and $G-V$ characteristics for nanowires with QPC formed between the Ge99.99 $\mathrm{Ga}_{0.01}$ surface and Co tip. There are typical curves, for which the positive threshold voltage value $V_{\mathrm{th}} \approx 3.1 \mathrm{~V}$ corresponding to the maximum of the histogram from Fig. 3.

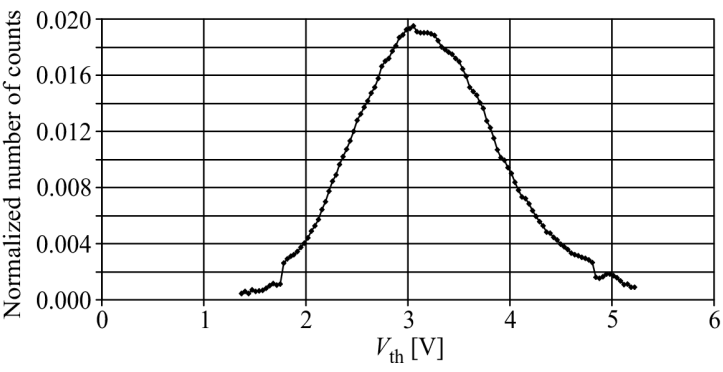

Fig. 3. The histogram for the threshold voltage $V_{\mathrm{th}}$ calculated on the basis of 1100 measurements of the current-voltage characteristic.

surement range) which causes an increase in the value of the current through the nanowire with QPC up to the value $I_{\min }$. The measurement is performed for the bias voltage $V$ in the range between points 2 and 3 . For this stage the voltage changes in the range from $V_{\min }$ to $V_{\max }$ during $2 \mathrm{~ms}$ period of time. The oscilloscope registers two voltage signals (82 samples per each) on basis of which values of voltage $V$ and current $I$ presented in Fig. 2a are calculated.

On the characteristics presented in Fig. 2 there are two different behaviors: (1) for the low bias the current $I$ (Fig. 2a) and conductance $G$ (Fig. 2b) increase slowly as a function of voltage $V,(2)$ the range after crossing the threshold voltage value $V_{\text {th }}$ the electric current as well as conductance rise rapidly. Rapid growth of the current and conductance for range (2) is caused by a strong electric field, which, in our opinion, induces the avalanche ionization causing exponential growth of the number of free carriers taking part in electric transport $[22,23]$. The value of the threshold voltage $V_{\text {th }}$ can de- pend on the length of the nanowire with QPC that is slightly different for each measurement. In Fig. 2 there are typical $I-V$ characteristics, for which the positive value $V_{\text {th }}$ equals approximately $3.1 \mathrm{~V}$. This value can be found from the histogram presented in Fig. 3. This histogram was calculated on the basis of 1100 measurements of the current-voltage characteristics. For each one of these characteristics a value $+V_{\text {th }}$ has been specified and on the basis of these results the histogram was calculated. The maximum of the histogram is at the value $V_{\text {th }} \approx 3.1 \mathrm{~V}$.

\section{Summary}

The presented method allows to measure the $I-V$ and $G-V$ characteristics for nanowires with QPC formed at the $\mathrm{Ge}_{99.99} \mathrm{Ga}_{0.01}$ interface. We use the $p$-type Ge sample which creates an ohmic contact with the metal independently of the metal work function. It allows us to measure electric transport properties of the nanowire without the need for compensation of the Schottky barrier effect, which usually occurs in the connection of the measuring probe with the surface of the semiconductors. Conducted measurements also confirmed that the Schottky barrier at the $\mathrm{Ge}_{99.99} \mathrm{Ga}_{0.01}$ interface under investigation does not occur. Due to the use of the doped semiconductor $\mathrm{Ge}_{99.99} \mathrm{Ga}_{0.01}$, resistivity of which equals about $\approx 4 \mathrm{~m} \Omega \mathrm{cm}$, resistance of Ge sample practically does not influence the nanowires conductance measurements. Characteristics presented in this work have shown the exponential growth of the current after crossing the threshold voltage value of the voltage. That effect can be useful in preparation of nanoelectronic devices. On the presented characteristics there is a slight asymmetry in respect of the voltage $V=0$. The reason for this effect is unclear and needs further investigation.

\section{Acknowledgment}

The authors are indebted to M. Szorcz for technical assistance by samples preparation.

\section{References}

[1] Y. Cui, C.M. Lieber, Science 291, 851 (2001).

[2] J.-R. Kim, H. Oh, H.M. So, J. Kim, J.-J. Kim, Physica $E$ 18, 225 (2003).

[3] M.-C. Jeong, B.-Y. Oh, M.-H. Ham, J.-M. Myoung, Appl. Phys. Lett. 88, 202105 (2006).

[4] P.-C. Chang, Z. Fan, C.-J. Chien, D. Stichtenoth, C. Ronning, J.G. Lua, Appl. Phys. Lett. 89, 133113 (2006).

[5] Z.Y. Fan, D.W. Wang, P.C. Chang, W.Y. Tseng, J.G. Lu, Appl. Phys. Lett. 85, 5923 (2004).

[6] Z.Y. Fan, J.G. Lu, Appl. Phys. Lett. 86, 123510 (2005).

[7] J.I. Pascual, J. Méndez, J. Gómez-Herrero, A.M. Baró, N. García, Vu Thien Binh, Phys. Rev. Lett. 71, 1852 (1993). 
[8] J.M. Krans, J.M. van Ruitenbeek, V.V. Fisun, I.K. Yanson, L.J. de Jongh, Nature 375, 767 (1995).

[9] M. Brandbyge, J. Schiotz, M.R. Sorensen, P. Stoltze, K.W. Jacobsen, J.K. Norskov, L. Olesen, E. Laegsgaard, I. Stensgaard, F. Besenbacher, Phys. Rev. B 52, 8499 (1995); K. Hansen, E. Lagsgaard, I. Stensgaard, F. Besenbacher, Phys. Rev. B 56, 2208 (1997).

[10] C.J. Muller, J.M. van Ruitenbeek, L.J. de Jongh, Physica C 191, 485 (1992).

[11] K. Hansen, S.K. Nielsen, E. Lagsgaard, I. Stensgaard, F. Besenbacher, Rev. Sci. Instrum. 71, 1793 (2000).

[12] J.L. Costa-Krämer, N. García, P. García-Mochales, P.A. Serena, M.I. Marqués, A. Correia, Phys. Rev. B 55, 5416 (1997).

[13] K. Hansen, S.K. Nielsen, M. Bradbyge, E. Lagsgaard, I. Stensgaard, F. Besenbacher, Appl. Phys. Lett. 77, 708 (2000).

[14] M. Yoshida, Y. Oshima, K. Takayanagi, Jpn. J. Appl. Phys. 44, L1178 (2005).
[15] M. Yoshida, Y. Oshima, K. Takayanagi, Appl. Phys. Lett. 87, 33 (2005).

[16] M. Wawrzyniak, J. Martinek, B. Susła, J. Phys., Conf. Ser. 146, 012035 (2009).

[17] A. Dimoulas, P. Tsipas, A. Sotiropoulos, E.K. Evangelou, Appl. Phys. Lett. 89, 252110 (2006).

[18] R.R. Lieten, S. Degroote, M. Kuijk, G. Borghs, Appl. Phys. Lett. 92, 022106 (2008).

[19] M. Wawrzyniak, Metrol. Measur. Syst. 14, 391 (2007).

[20] T. Nishimura, K. Kita, A. Toriumi, Appl. Phys. Lett. 91, 123123 (2007).

[21] M. Wawrzyniak, Metrol. Measur. Syst. 13, 161 (2006).

[22] D. Wang, J.G. Lu, C.J. Otten, W.E. Buhro, Appl. Phys. Lett. 83, 5280 (2003).

[23] D.-I. Kim, N. Pradeep, F.W. DelRio, R.F. Cook, Appl. Phys. Lett. 93, 203102 (2008). 